8. NSW Department of Health, Public Health Training and Development Unit. Informing public health practice competencies of the graduate diploma of applied epidemiology. State Health Publication No: (PHD) 000046. Sydney: NSW Department of Health, 2000.

9. Dolmans DHJM, DeGrave W, Wolfhagen IHAP, Van der Vleuten CPM. Problem-based learning: Future challenges for educational practice and research. Med Educ 2005; 39(7): 732-41. doi:10.1111/j.1365-2929.2005.02205.x

10. Nesbit T. Mapping adult education. Educ Theory 1999; 49(2): 265-79. doi:10.1111/j.1741-5446.1999.00265.x

11. Albanese M, Mitchell S. Problem-based learning: a review of literature on its outcomes and implementation issues. Acad Med 1993; 68(1): 52-81. doi:10.1097/00001888-19930100000012
12. Berkson L. Problem-based learning: have the expectations been met? Acad Med 1993; 68(Suppl): S79-88. doi:10.1097/00001888-199310000-00053

13. Vernon D, Blake R. Does problem-based learning work? A meta-analysis of evaluative research. Acad Med 1993; 68: 550-63. doi:10.1097/00001888-199307000-00015

14. Rogers C. Learning theories: Experiential learning. Available at http://www.educationau.edu.au/archives/cp/04f.htm. Accessed 24 February 2006.

15. Conner ML, Wright KC, DeVries L, Zeider C, Wilmsmeyer D, Forman D. Learning: the critical technology. Missouri: Wave Technologies International Inc., 1996. Available at www.wavetech.com/abt/abttmwp.htm. Accessed 1 August 2006.

\title{
Use of communication technology among public health professionals in New South Wales, Australia
}

\section{Carlie-Jane Naylor ${ }^{\mathrm{A}, \mathrm{C}}$, D. Lynne Madden ${ }^{\mathrm{A}}$ and Deborah J. Oong ${ }^{\mathrm{B}}$}

APublic Health Training and Development Branch, NSW Department of Health

${ }^{\mathrm{B}}$ Corporate Governance and Risk Management Branch, NSW Department of Health

${ }^{\mathrm{C}}$ Corresponding author.Email: carlie.naylor@doh.health.nsw.gov.au

\section{Introduction}

Communication technology plays an important role in health services in NSW, not only for the delivery of clinical services but also as a means of delivering training, holding meetings and sustaining networks. One reason for this is the large geographical size of NSW; it is divided into eight area health services, the largest of which covers $444586 \mathrm{~km}^{2}{ }^{1}$ Communication technology provides a means of linking health professionals across areas, and is particularly useful for those working in rural and remote communities. Even where distances are not large, these techniques also allow a more efficient use of time by reducing the need for travel.

Teleconferencing is frequently used for different types of meetings because it is readily available and easy to use. These features led Wildsoet et al. ${ }^{2}$ to pilot a continuing eduction program via teleconferencing to optometrists in rural Queensland. There are, however, limitations to teleconferencing. A randomised controlled trial compared teleconferencing with videoconferencing for the delivery of multidisciplinary case conferences. ${ }^{3}$ The trial found that there were more benefits with videoconferencing, and a small survey conducted with trial participants highlighted that having a visual component generated a high level of satisfaction in team members.

Videoconferencing is widely used in medicine for delivering clinical services such as mental health. ${ }^{4}$ In these clini- 
Table 1. Six communication techniques used in the survey of the public health workforce

\begin{tabular}{ll}
\hline Technique & Description \\
\hline $\begin{array}{l}\text { Teleconference } \\
\text { Web bulletin boards }\end{array}$ & $\begin{array}{l}\text { Interactive exchange of voice, usually of more than two participants, by telephone } \\
\text { Eeb conference }\end{array}$ \\
$\begin{array}{l}\text { Conducted over the Internet between two or more people situated at different locations; enables } \\
\text { videos, websites, PowerPoint presentations and software demonstrations to be displayed in real time to } \\
\text { all participants }\end{array}$ \\
$\begin{array}{l}\text { Tideoconference } \\
\text { Media streaming }\end{array}$ & $\begin{array}{l}\text { Transmission of video with synchronised sound in compressed form over the Internet to be viewed as a } \\
\text { continuous stream }\end{array}$ \\
Satellite television & A live broadcast over a satellite network \\
\hline
\end{tabular}

cal settings, there are two sites: one where the clinician is based and the other a remote site where the patient is located. This type of videoconference is referred to as point-to-point. Videoconferencing can also be used to deliver continuing professional development activities to multiple remote sites while retaining an environment that supports collegiate networking. ${ }^{5}$

Streaming media over the Internet provides a solution when training schedules conflict with other commitments. Chong et al. described their experience of setting up and delivering presentations by media streaming to pharmacy staff at a Vancouver hospital. Staff who were unable to attend the face-to-face sessions were able to access the presentations at their own convenience, either on their work or home computer. ${ }^{6}$ Other computer-based applications, such as web conferencing and web bulletin boards, offer alternative ways to support the delivery of training.

Another effective medium for delivering continuing education to large groups is satellite television. The Centers for Disease Control and Prevention (CDC) have used this medium to deliver courses to multiple sites across the United States. ${ }^{7,8}$

There may be opportunities to expand the contribution of communication technology to public health work. In order to inform strategic planning for the public health workforce and identify opportunities to use communication technology, a survey was undertaken to provide a 'snapshot' of the current use of communication techniques by public health professionals. The forms used, the situations in which these are applied and the level of use were explored.

\section{Methods}

Thirteen senior public health professionals working within the NSW Department of Health and/or area health services were invited to participate in the survey. Participants from the area health services were chosen from the population health structures, including health promotion units and public health units. A community health perspective was also sought. Three participants were drawn from the Division of Population Health of the NSW Department of Health. Two of the 13 public health professionals who were invited to participate were drawn from rural areas and the remainder from metropolitan areas.

A three-part, structured questionnaire with closed and open-ended questions was designed for the survey. The first part sought characteristics of the participants and their work. As two of the most common applications of communication technology are to conduct meetings and to deliver professional development activities, estimates of the amount of time spent in these activities were sought. The second part asked about the participants' experience of using six techniques, teleconferencing, web bulletin boards, web conferencing, videoconferencing, media streaming and satellite television, and their interest in expanding their experience. The third part explored factors that would prevent or assist them in using these techniques.

The interviews were conducted in March and April 2005. The questionnaire was administered to the participants by the same interviewer, either in person or over the telephone. Before commencing the interview with each participant, the interviewer established the context and the scope for the interview with a scripted preamble. Recognising that not all the participants would be familiar with the techniques being investigated, the interviewer used standardised descriptions of each type of communication technique (Table 1). The survey and preamble were piloted with a public health professional, and the feedback was used to amend the questionnaire and its delivery.

Responses were entered into a database created in Epi Info version 3.3 (CDC, Atlanta, GA, USA). For the closed questions, frequencies were reported. A thematic analysis was carried out on the responses to the open-ended questions. 


\section{Results}

\section{Characteristics of the participants}

Twelve of the 13 public health professionals agreed to be interviewed (response rate, 92\%). The roles and responsibilities of the participants spanned managing units or teams; leading population health and community services; development of evidence-based guidelines and policies; workforce development; facilitating health promotion; and the provision of population health services. The number of people reporting to individual participants varied from one full-time equivalent position to about 1000 (through direct and indirect reporting lines).

The amount of participants' time taken up with meetings and/or delivering professional development activities ranged from 20 to $80 \%$. The amount of time that participants reported they spent travelling to these activities varied from 'very little', with meetings being held at their worksite, to about 2 hours a day. Five participants indicated that the amount of time dedicated to travelling to meet with staff and fulfil other organisational functions was likely to increase as a result of the restructuring of health services in NSW.

\section{Experience of communication technology}

All 12 participants reported using teleconferencing and 11 were familiar with videoconferencing. The three computer-based modalities and satellite television had been used by only a few participants.

The techniques were most commonly used for continuing professional development, meetings, interview panels and collaboration. Videoconferencing and teleconferencing were used for the widest range of activities, whereas web bulletin boards were primarily used for disseminating information to staff. While a couple of individuals used a wide range of techniques, no individual used all forms.

Circumstances often determined when the technology was used. For example, teleconferencing was used when the distance to travel for a face-to-face meeting was prohibitive or when an urgent issue had to be addressed at short notice. One participant, who described face-to-face meetings as the 'ideal', considered that videoconferencing provided a way to meet in real time when that ideal was not possible.

\section{Barriers and enabling factors}

Enabling factors that promoted the use of these techniques were: ease of access to facilities; assistance in organising and setting up the technology; the perception that the technology allowed efficient and effective use of time; and suitability of the technique for the intended purpose. Communication technology was viewed as a potentially cost-effective way of providing training opportunities for staff and limiting the need to travel.
The barriers to using these communication techniques were: difficulty in accessing the equipment; perceived difficulty in setting up and operating the equipment; and cost of use. Participants considered that skill and expertise are required to use these techniques successfully. They also reported having limited knowledge about use of many of them and lack of familiarity with the variety of techniques potentially available and their applications.

Nine participants indicated that there were opportunities to expand use of these techniques in the workplace. Suggestions included the delivery of training, the dissemination of information, working collaboratively, and exploring ways of combining use of these techniques with face-to-face delivery.

\section{Discussion}

Two techniques, teleconferencing and videoconferencing, are well established in practice. All participants used teleconferencing, and most used it frequently in everyday practice as it was seen as being easy to use and accessible. The advantage of videoconferencing over teleconferencing was the opportunity for personal contact and visual cues, features that were valued when higher levels of engagement were required. Satellite television was reserved for continuing professional development activities with large groups because of the costs associated with its delivery.

This survey was carried out with a small, selected group of public health professionals, so while the findings provide an insight into the current use of communication technology across a broad range of pubic health practice, they are indicative only. Further, the survey asked about current and potential use of these techniques. Information about the difficulties being experienced in their use was not sought. Consequently, the nature of the questions might have shaped both the information collected and the positive response of the participants.

Generally, the participants used what was familiar to them or had been recommended by colleagues. Having knowledge about and skills in the use of a technique appeared to lead to its routine application. However, familiarity alone did not determine whether a technique was used and a judgement was made about whether it was appropriate for the task. The efficient use of time and positive outcomes from the experience encouraged future use. Conversely, poor-quality interactions were discouraging, particularly when there was a choice for how a task was managed. Participants were clear about the factors that helped them to engage with these techniques, and they were discerning in their use.

Many participants raised cost as an issue, inferring that techniques that were cheap were more likely to be used. 
However, there can be a trade-off between cost and quality of delivery. For example, for videoconferencing, better quality transmission is associated with higher cost and cheaper options might be a false economy. There are nevertheless potential savings to be gained by the health system in using communication technology, in particular to reduce costs associated with travel.

Use of these techniques expands the size of the population that can potentially participate in activities, for example, meetings, training and mentoring, providing opportunities that would otherwise not have been available. For many of these individuals, face-to-face attendance at these events is not an option so their participation through the use of communication technology may present an additional cost to the system.

A review of the NSW Public Health Network presented in 2006 suggested that greater use could be made of communication technology to maintain collegiate networks and deliver public health functions. ${ }^{9}$ A response to this prepared by Directors of Public Health in NSW acknowledged increased reliance on communication technology while identifying many of the barriers to use described in this survey. In addition to those barriers that exist at the organisation level, effort is also required by individuals to change the way they use communication technology.

To encourage use of these techniques, ways to minimise the barriers and ensure high-quality interactions should be explored. As teleconferencing and videoconferencing are the most commonly used techniques, a priority should be to ensure that they are used effectively to support public health functions.

\section{Conclusion}

This sample of population health professionals engaged enthusiastically in considering how use of communication technology might be expanded to support population health work. These techniques enable remote collegial networks and continuing professional development. Information from this survey was used as a basis for a sub- mission by the Division of Population Health for Telehealth funding in 2005-06. A report of this survey, including the questionnaire, will be published as a supplement to the NSW Public Health Bulletin.

\section{Acknowledgements}

We would like to thank the participants of this survey. We would also like to thank the anonymous reviewers and Elisabeth Heseltine for their comments and suggestions on ways to improve this manuscript.

\section{References}

1. Greater Western Area Health Service. Greater Western Area Health Service Annual Report 2004/05. Available at www.gwahs.nsw.gov.au/files/AReport_Web_Version.pdf. Accessed 18 March 2007.

2. Wildsoet C, Wood J, Parke J. Audio-teleconferencing as a medium for distance learning: its application for continuing education in optometry. Aust J Rural Health 1996; 4(1): 18-27.

3. Wilson SF, Marks R, Collins N, Warner B, Frick L. Benefits of multidisciplinary case conferencing using audiovisual compared with telephone communication: a randomized controlled trial. J Telemed Telecare 2004; 10(6): 351-4. doi:10.1258/1357633042602026

4. Dossetor DR, Nunn KP, Fairley M, Eggleton D. A child and adolescent psychiatric outreach service for rural New South Wales: a telemedicine pilot study. J Paediatr Child Health 1999; 35(6): 525-9. doi:10.1046/j.1440-1754.1999.00410.x

5. Madden DL, Naylor CJ, Simpson D. An evaluation of videoconferencing Bug Breakfast. NSW Public Health Bull 2003; 14: S-2.

6. Chong E, Balen RM, Jewesson PJ. Delivery of multimedia educational presentations via streaming media. Am J Health Syst Pharm 2003; 60(23): 2475-8.

7. Maetz HM, Walton W, Smith M, Lincoln R, Galvin M, Tryon C et al. "A satellite primer on tuberculosis": a collaboration in distance education. J Public Health Manag Pract 1998; 4(5): 46-55.

8. Umble KE, Cervero RM, Yang B, Atkinson WL. Effects of traditional classroom and distance continuing education: a theory-driven evaluation of a vaccine-preventable diseases course. Am J Public Health 2000; 90(8): 1218-24.

9. Morey S. Review of the NSW Public Health Network. Final report. [Internal NSW Health Report]. Morey Australia, March 2006. 